\title{
Intertextualité et réécriture à travers "Métamorphoses de la reine / de Pierrette Fleutiaux
}

\author{
Recherche présentée par \\ Mennatallah Mohamad Tawfik \\ Assistante au département de français \\ Faculté des lettres \\ Université de Mansoura
}


Q'est-ce à dire intertextualité? Est-ce qu'il y a de différence entre hypertextualité et intertextualité ? Quels sont les procédés à suivre pour dire que tel ou tel texte est en relation intertextuelle avec un autre ? A vrai dire, l'intertextualité et l'hypertextualité sont deux notions qui ne peuvent pas être étudiées séparément. Au contraire, elles s'interfèrent et s'entrelacent. Les deux termes signifient qu'un texte est en relation avec un autre qui lui est antérieur, et cela peut se faire d'une manière ou d'autre. D'ailleurs, certains analystes considèrent que «la réécriture est un cas particulier d'intertextualité. »"

Il est à ajouter que l'intertextualité est à la base de toute création littéraire. Un texte ne peut pas se faire en partant de rien :

"Nul texte ne peut s'écrire indépendamment de ce qui a été déjà écrit et il porte, de manière plus ou moins visible, la trace et, la mémoire d'un héritage et de la tradition. L'intertextualité serait alors, peut-être simplement et banalement, le fait que toute écriture se situe toujours parmi les ouvres qui la précèdent et qu'il n'est jamais possible de faire table rase de la littérature. »²

(1) Anne-Claire Gignoux, La récriture: formes, enjeux, valeus autour du nouveau roman, éd., Presses Paris Sorbonne, 2003,p.15.

(2) Nathalie Piégay-Gros ,Introduction à l'intertextualité, éd., Dunod, Paris,1996,p.7.

Vol. 39 
Intertextualité et réécriture à travers « Métamorphoses de la reine » de Pierrette Fleutiaux

Sur ce, l'intertextualité est une pratique qui existe depuis longtemps. Elle signifie l'interaction et les échanges entre les textes comme l'indique son étymologie*. Ce qui peut faire de l'intertextualité une pratique illimitée. Prenons l'âge classique par exemple, où l'imitation de la tradition grécolatine était l'idéal dans le domaine littéraire pour la plupart des écrivains à l'époque. Citons la Fontaine disant à ce sujet :

"On s'égare en voulant tenir d'autres chemins. "

Donc, l'intertextualité était une partie adhérente à la littérature. Même si elle n'était pas étudiée, définie et analysée depuis longtemps, elle était pratiquée en tout cas. Il fallait attendre les années soixante du siècle dernier pour attester la théorisation et la conceptualisation du terme. Et il est à signaler que cette pratique étant vaste et riche, continue à être étudiée jusqu'à nos jours.

\footnotetext{
*D'après le préfixe latin "inter" on comprend l'idée de la réciprocité des échanges, l'interconnexion, l'interférence. Le radical latin 'textere' 'textualité' évoque la qualité du texte comme 'tissage', 'trame'. Hans-George Ruprecht, "Intertextualité » texte (Toronto), ${ }^{\circ} 2$ 2,1983,p.13-15 ( Républication d'une notice également prévue pour le Dictionnaire international des termes littéraires), in "L'intertextualité », textes réunis par Nathalie Limat-Letellier et Marie MiguetOllagnier, Presses univ. Franche-Comté,p.17.*

${ }^{(1)}$ Encyclopédie Larousse en ligne. www.larousse.fr/encyclopedie.

Vol. 39

January 2015
} 
Le théoricien Mikhaïl Bakhtine était le premier à introduire la notion du «dialogisme» dans la littérature. Il voyait qu'un mot peut entamer un dialogue entre le texte et son lecteur, mais aussi entre les textes eux-mêmes. En s'appuyant sur son étude, c'est Julia Kristeva qui a utilisé le terme « intertextualité ». Elle le définit comme :

«Croisement dans un texte d'énoncés pris à d'autres textes et transposition d'énoncés antérieurs ou synchroniques. ${ }^{l}$

Nous rencontrons maintes études au sujet de l'intertextualité. Barthes, Laurent Jenny, Michael Riffaterre sont aussi des théoriciens de l'intertextualité. Nous retrouvons aussi le terme "vertige »" employé par Michel Schneider pour qualifier les jeux intertextuels. Mais comme la plupart des études ont beaucoup de points en commun et traitent les mêmes côtés de l'intertexte mais différemment,passons directement à l'étude «Palimpsestes».

(1) Julia Kristeva, Sémiotiké, Recherche pour une sémanalyse, in Intertextualité mémoire de la littérature, Tiphaine Samoyault, éd., Nathan, Paris, 2001,p.9.

*Il dit «l'espace littéraire est un espace régi par un vertige essentiel. Chaque livre est l'écho de ceux qui l'anticipèrent ou le présage de ceux qui le répéteront. » Michel Schneider, Voleurs de mots, Paris, Gallimard, 1985,p.81. *

Vol. 39 
Intertextualité et réécriture à travers « Métamorphoses de la reine » de Pierrette Fleutiaux

Genette nous donne une définition un peu restreint de l'intertextualité. Il la qualifie de :

«Une relation de coprésence entre deux ou plusieurs textes, c'est-à-dire, éidétiquement et le plus souvent, par la présence effective d'un texte dans un autre. Sous sa forme la plus explicite et la plus littérale,c'est la pratique traditionnelle de la citation ( avec guillemets, avec ou sans référence précise); sous une forme moins explicite et moins canonique, celle du plagiat (...) qui est un emprunt non déclaré,mais encore littéral,sous forme encore moins explicite et moins littérale, celle de l'allusion, c'est-à-dire d'un énoncé dont la pleine intelligence suppose la perception d'un rapport entre lui et un autre auquel renvoie nécessairement telle ou telle de ses inflexions... »l

L'intertextualité, selon Genette, signifie simplement la présence d'un texte dans un autre texte, et cela peut se faire avec des nuances. Il indique trois types de ces pratiques à savoir : la citation, l'allusion et le plagiat. La dernière pratique consiste à s'approprier les travaux ou les idées des autres et les présenter comme siens, sans en citer la source. Certains écrivains célèbres ont été accusés de plagiat comme Calixthe Beyala et Michel

${ }^{(1)}$ Gérard Genette, Palimpsestes,op.cit.,p8. Vol. 39 
Houelbecq, mais, heureusement, cela n'est pas le cas chez notre écrivaine. Comme l'écriture ou la réécriture de Fleutiaux s'émane de sa propre imagination, son texte est apparu comme création neuve et pertinente. En s'appuyant sur les nouvelles à étudier, notre analyse sera balisée sur deux points : la citation et la référence et chacune a certes ses enjeux et ses procédés.

En appréhendant le champ intertextuel dans le livre de Fleutiaux, il y aura certains points à étudier, et ils nous semblent être au nombre de quatre :

1- L'intertextualité au niveau paratextuel

2- L'intertextualité au niveau textuel

3- L'intertextualité avec l'hypotexte

4- Les fonctions de l'intertextualité

Dans le chapitre consacré à la paratextualité, nous avons étudié en détail les éléments de paratexte dans le texte fleutien et l'influence de la réécriture sur eux, tout en dressant une comparaison entre l'hypotexte perraldien et l'hypertexte de Fleutiaux. Ces éléments seront réétudiés ici mais sous un angle d'approche différent. Pour cerner la notion d'intertextualité dans le paratexte nous allons étudier : le titre, la préface, les intertitres, les notes et la quatrième de 
Intertextualité et réécriture à travers « Métamorphoses de la reine » de Pierrette Fleutiaux couverture. C'est-à-dire les lieux où on peut rencontrer les empreintes de l'intertexte.

\section{a-La citation}

C'est la forme canonique de la typographie de l'intertextualité introduit par Genette. Elle est aussi un type explicite de l'intertexte. Antoine Compagnon dans son étude majeure sur la citation, la compare à la « greffe » :

«La citation est un corps étranger dans mon texte, parce qu'elle ne m'appartient pas en propre, parce que je me l'approprie. Aussi son assimilation, de même que la greffe d'un organe, comporte-t-elle un risque de rejet contre lequel il faut me prémunir et dont l'évitement est l'occasion d'une jubilation. » ${ }^{1}$

Là-dessus, il faut bien choisir la citation avant de l'insérer dans mon texte, sinon elle sera comme une sorte de discordance qui interrompt l'unité et la linéarité du texte sans aucun intérêt.

Pour en revenir à notre corpus, les pratiques intertextuelles dans l'œuvre de Fleutiaux peuvent s'appliquer sur plusieurs

${ }^{(1)}$ Antoine Compagnon, La seconde main ou le travail de la citation, éd. du Seuil, Paris, 1979,p.31.

Vol. 39 
parties du texte, mais aussi du paratexte. Dans son œuvre, l'intertexte au niveau paratextuel existe à travers certains éléments comme nous allons voir au fur et à mesure de l'analyse.

La citation est marquée par l'italique dans la plupart de temps, ou bien elle est mise entre deux guillemets qui soulignent que c'est un extrait d'une œuvre autre. Elle est souvent accompagnée d'une référence qui en indique la source. Mais chez Fleutiaux, le cas est un peu différent.

Commençons par «Cendron » qui a eu la plus grande part de citations. Dans cette nouvelle on rencontre une citation mise entre guillemets, et deux autres qui sont citées sans référence aucune. Mais cela ne peut pas être considéré comme plagiat puisque les textes cités sont extraits des chefs d'œuvre de la littérature française et qui sont assez célèbres et connus de la part de destinataires.

Le nouveau héros du conte «Cendron » est allé au bal qu'avait organisé la reine pour choisir l'un des prétendants pour se marier avec sa fille. En arrivant à la fête, il ignore la princesse qu'il a trouvé superficielle et frivole. A ce momentlà, elle s'adresse aux deux frères de Cendron qui étaient 
Intertextualité et réécriture à travers « Métamorphoses de la reine » de Pierrette Fleutiaux invités, eux aussi, à cette fête : le boiteux et l'autre qui tousse tout le temps, pour l'embarrasser et l'exaspérer, elle leur dit :

«-Allons, dit la princesse, si votre plumage se rapporte à votre ramage, baisez-moi fort promptement. ${ }^{1}$

Ici elle cite la Fontaine dans sa célèbre fable « $L e$ corbeau et le renard», dont la moralité porte sur la flaterie exagérée des hypocrites, mais en inversant les vers originaux :

«Sans mentir,si votre ramage

Se rapporte à votre plumage,

Vous êtes le Phénix des hôtes de ce bois. »²

Cet inversement peut avoir un effet d'ironie et elle s'adapte aussi au nouveau contexte. Les deux frères ne font rien sauf parler sans action. La princesse se moque des deux jeunes hommes maladroits, en essayant d'attirer l'attention de Cendron qui se moque de son attitude légère. Mais il est à signaler que cette phrase est absorbée et intégrée dans le trame du texte sans guillemets, sans référence ni nom d'auteur. Ce type de citation est appelé «impli-citation », qui désigne «la

\footnotetext{
${ }^{(1)}$ Pierrette Fleutiaux, Métamorphoses de la reine,op.cit.,p.69.

${ }^{(2)} \mathrm{www}$.jdlf.com/lesfables/livres.
}

Vol. 39 
citation implicite, entièrement fondue dans le texte d'accueil. " ${ }^{1}$

Plus précisément, c'est une impli-citation simple, c'està-dire qu'elle supprime ou dissimule toute sorte de signes de changement d'énonciation. Ainsi, l'énonciateur s'attribue les dits des autres d'une manière directe et nette. ${ }^{2}$

Contrairement aux autres types de citations, l'impli-citation (abréviation d'implicite, bien entendu) exige plus d'effort de celui qui lit afin de désigner la citation et l'interpréter, ce qui dépend de sa culture et ses connaissances littéraires.

Sous la même catégorie, vient s'ajouter une autre citation, cette fois-ci appartenant à Molière. Dans son «Bourgeois gentilhomme» Molière nous présente le protagoniste de sa pièce, Monsieur Jourdain, qui essaye d'apprendre plusieurs manières «galantes» pour écrire une lettre ou un billet d'amour. Ici , il engendre un effet comique en composant la même phrase avec les mêmes mots mais en désordre. Nous proposons une partie de la pièce qu'est un dialogue entre M. Jourdain et le maître de philosophie :

\footnotetext{
${ }^{(1)}$ Tiphaine Samoyault, L'intertextualité,op.cit.,p.44.

(2) Ibid.,p45.
}

Vol. 39 
Intertextualité et réécriture à travers «Métamorphoses de la reine » de Pierrette Fleutiaux

«-M.Jourdain : par ma foi ! il y a plus de quarante ans que je dis de la prose sans que j'en susse rien, et je vous suis le plus obligé du monde de m'avoir appris cela. Je voudrais donc lui mettre dans un billet : "Belle marquise, vos beaux yeux me font mourir d'amour », mais je voudrais que cela fût mis d'une manière galante, que ce fût tourné gentiment.

\section{$-(\ldots)$}

-Maître de philosophie : on les peut mettre premièrment comme vous avez dit: "Belle marquise, vos beaux yeux me font mourir d'amour. " ou bien : "d'amour mourir me font, belle marquise, vos beaux yeux » ou bien : "vos yeux beaux d'amour me font, belle marquise, mourir» ou bien : «mourir ». ${ }^{1}$

Ce procédé rhétorique, l'anastrophe, qui consiste à renverser l'ordre habituel ou grammaticale des mots, est repris par Fleutiaux. Cendron, comme Cendrillon, a fuit le bal à minuit afin que l'enchantement des ses vêtements et de sa Cadillac ne disparaîsse pas. Mais dans la réécriture l'heure fatidique est annoncée par un talkie-walkie qu'il a laissé tombé en prenant la fuite, au lieu de la pantoufle de

\footnotetext{
${ }^{(1)}$ Molière, «Le bourgeois gentilhomme », acte II,scène IV, cf.www.sitemoliere.com/pieces/bourg.204.htm.

Vol. 39 
Cendrillon. L'envoyé de la reine présente cette boîte à tous les hommes du royaume pour savoir qui est son propriétaire original. Il la présente encore aux deux frères de Cendron. Chacun prononce un compliment, une phrase devant cette «boite noire ». Le premier l'approche de sa bouche en disant :

«les beaux yeux de la princesse d'amour me font mourir. ${ }^{l}$

L'autre reprend à peu près les mêmes mots :

«D'amour mourir vos beaux yeux princesse me font. »"

Elle s'inspire cette fois-ci des pièces classiques de Molière peut être pour donner un aspect drôle aux frères de Cendron qui essayent de l'imiter en montrant qu'ils sont cultivés, tandis qu'ils ne savent rien du tout. Le second frère répète la parole de l'autre puisqu'il n'a rien à ajouter. Ces deux phrases relèvent tout de même de l'impli-citation dont on vient de parler. Elles sont tirées du registre littéraire sans marquer leur source.

Dans une autre partie de la même nouvelle, nous rencontrons un autre extrait d'une œuvre qui remonte au $14^{\mathrm{e}}$

\footnotetext{
${ }^{(1)}$ Pierrette Fleutiaux, Métamorphoses de la reine,op.cit.,p.77.

(2) Ibid.
}

Vol. 39 
Intertextualité et réécriture à travers « Métamorphoses de la reine » de Pierrette Fleutiaux siècle, celle de Dante intitulée «La divine comédie ». ${ }^{1}$

En essayant de s'éloigner du bal, Cendron entre dans le palais et rencontre la reine par hasard, dans sa bibliothèque :

"Ils arrivèrent à l'alcôve aux petits bouts de chandelle,la reine en alluma une et chercha dans ses livres. Lorsqu'elle en eût sorti un, et avant, même qu'elle l'eût ouvert, Cendron récita ces mots : "Je me trouvais au milieu de ma vie dans une sombre forêt... ${ }^{2}$

Dans son texte original, Dante disait :

«Sur le milieu du chemin de la vie

Je me trouvai dans une forêt sombre. ${ }^{3}$

C'est comme cela qu'il a commencé chant I de son poème, cette partie qui portait le titre : "l'enfer ».

Contrairement à la phrase de Molière empruntée par ses frères, Cendron cite Dante ici d'une manière correcte, exactement comme elle a été écrite dans l'œuvre originale,

${ }^{(1)}$ Poème épique et allégorique composé par l'italien Dante Alighieri entre 1307 et 1321. Il est composé de 3 parties : "L'enfer », "Le purgatoire »,et "Le paradis », chacune est composée à son tour de 33 chants. Il est considéré comme le chefd'œuvre de la littérature médiévale. Cf.www.wikipedia.org.

${ }^{(2)}$ Pierrette Fleutiaux,Métamorphoses de la reine,op.cit.,p.74.

${ }^{(3)}$ www.operone.de/dante.

Vol. 39 
comme signe de culture et d'érudition. Et pourqoui pas ? Il passait des nuits à lire des livres et des manuels dans toutes les spécialités que lui avait légués son père. Lorsqu'il récite ces vers, cela nous montre qu'il connaît ce livre par cœur, contrairement à ses frères qui prononcent des phrases qu'ils avaient probablement entendues quelque part! L'auteur par cette citation nous donne un trait de son caractère, mais aussi elle met en relief sa propre connaissance en tant qu'écrivaine. Peut être elle a eu recours aux guillemets dans cette citation pour faire un clin d'œil au lecteur ordinaire que c'est la parole de quelqu'un d'autre. Elle lui dit « je cite » parce que ce texte peut être connu par certains lecteurs seulement. Ces guillemets peuvent pousser le lecteur soit à interrompre sa lecture pour trouver la référence et ainsi elle casse la linéarité du texte, soit à continuer normalement sa lecture, et il est à lui de choisir.

Passons maintenant à d'autres exemples de l'intertextualité mais cette fois-ci ils sont tirés des éléments paratextuels. D'abord, les notes «notules» prennent leur place dans le recueil à la fin de la nouvelle «Petit Pantalon Rouge». Dans cette partie nous pourrons signaler deux citations. 
Intertextualité et réécriture à travers « Métamorphoses de la reine » de Pierrette Fleutiaux

La première citation est mentionnée comme explicaiton du discours du loup qui, ne pouvant pas parler après avoir avaler la gomme qui colle tout, commence à prononcer des mots incompréhensibles : «psr», «иии »,.... Fleutiaux essaye de nous expliquer ces mots de plusieurs façons. La citaiton ici n'est pas française, mais une phrase en latin qu'est «veni,vidi,vici ». ${ }^{l}$ Une célèbre phrase employée par Jules César.

Bien sûr cette expression est utilisée dans un dessein ironique dans l'intention de mépriser le loup « vaincu » ! Il est à mentionner que cette formule a été reprise dans plusieurs œuvres artistiques surtout dans le domaine de la musique. Mais elle a été aussi détournée. Fleutiaux, elle-même la cite de deux autres manières en essayant d'interpréter les paroles du loup, mais tout en gardant la division de la formule de César, et en respectant son rythme ternaire ; ainsi le loup aurait pu dire :

«Elle pue,elle sue,elle rue. » ${ }^{2}$

Ou bien :

\footnotetext{
${ }^{(1)}$ Pierrette Fleutiaux , Métamorphoses ,op.cit.,p.137.

(2) Ibid.,p.136.
}

Vol. 39 
« Je lai vue, je l'ai eue,je m'en fus. »"

La même citation a été donc reprise dans la même partie du texte mais elle a été détournée, ou réécrite. Cela relève du style ironique qu'adopte l'écrivaine.

Nous comprenons que l'intertexte ne se restreint pas au domaine littéraire, au contraire il s'étend sur d'autres domaines. Par exemple si l'on trouve dans un roman ou un recueil un extrait de presse, une ordonance ou même une recette de cuisine, tout cela vient s'organiser sous le titre intertextualité. Cela s'applique sur la phrase cité par Fleutiaux, par laquelle elle termine ses notes. Elle interprète ainsi le discours de la fumée :

"Quel bel homme,quel bon parti,et tout à votre service. $»^{2}$

La première partie de ce discours peut former une relation intertextuelle avec la parole d'un politicien célèbre qui s'appelle Jack Lang.*

${ }^{(1)}$ Ibid.

${ }^{(2)}$ Métamorphoses de la reine,p.138.

*Jack lang (1939- ) homme politique français qui a été nommé ministre à plusieurs mandats. Il était ministre de la culture et de l'éducation nationale. Vol. 39 
Intertextualité et réécriture à travers « Métamorphoses de la reine » de Pierrette Fleutiaux

Cet homme occupait le poste de ministre de la culture en 1981 et pendant dix ans, c'est-à-dire lors de l'écriture du recueil sujet de notre étude. Jack Lang a été la cible de Laurent Gerra, un humoriste et imitateur, à cause de cette phrase «Quel bel homme»!

Nous lisons dans un article publié dans l'hebdomadaire français «Le point» ce qui montre que ce « satiriste » essaye toujours de saisir les phrases tant répétées par un chanteur, un acteur ou un homme politique, ou bien une attitude, ou un trait de personnalité qui le marque pour essayer de l'imiter d'une manière subtile. Et l'auteur de cet article nous montre que Jack Lang s'exclame à tout propos «quel bel homme! " . Il se peut que l'écrivaine, tout en conservant son ton d'humour, a cité cette phrase comme signe de moquerie des paroles du ministre, ou bien pour le critiquer.

Sur la quatrième de couverture, on remarque aussi une citation de Bertrand Poirot-Delpech*, c'est plutôt son opinion

Maintenant il est le président de l'institut du monde arabe à Paris. Cf.www.wikipedia.org. *

${ }^{(1)}$ Le point.fr, article publié le 27/06/1998, par Sophie Coignard.

*Bertrand Poirot-Delpech (1929-2006 à Paris), journaliste, essayiste et romancier français, membre à l'Académie française de 1986 à sa mort. Il a travaillé notamment pour le quotidien « Le Monde ». cf.www.wikipedia.org. *

Vol. 39 
publiée dans l'un des journaux français à propos du recueil de Fleutiaux :

«A force d'intelligence joueuse, de violence sensuelle et de confusion suggestive entre les mots et les choses, Pierrette Fleutiaux mérite qu'on dise désormais d'elle: Il était une fois un maître du fantastique. " ${ }^{1}$

Cette citation a été bien choisie, elle occupe sa place adéquate sur la couverture. On peut dire qu'elle remplit deux fonctions: d'abord, c'est une sorte d'éloge qui montre que c'était un livre bien accueilli de la part des critiques, ce qui peut, à son tour, encourager un lecteur probable à en acheter un exemplaire. Et deuxièmement, en utilisant la célèbre formule des contes «Il était une fois » il peut accrocher l'attention du lecteur et montrer qu'il est en relation avec ces contes. Ils s'inspire d'eux, il les reprend d'une façon tout à fait nouvelle.

La pratique de la citation a été utilisée par Fleutiaux à maintes reprises. Tantôt elle recourt à l'héritage littéraire français, et tantôt elle cite des phrases en d'autres langues. Tout de même, elle reprend des phrases non-littéraires (qui viennent du domaine de la politique par exemple) comme 
Intertextualité et réécriture à travers «Métamorphoses de la reine » de Pierrette Fleutiaux nous venons de voir. Mais enfin, la citation reste le procédé intertextuel le plus utilisé chez elle.

\section{b- La référence}

Une autre forme explicite de l'intertextualité, c'est la référence. Cette forme n'a pas été étudiée par Genette, donc nous nous appuyons sur d'autres théoriciens qui ont analysé ce point. D'après cette pratique, on se renvoie à un texte antérieur sans le citer explicitement, ou littéralement. Cela va dans le même sens des mots de Tiphaine Samoyault :

«la référence n'expose pas le texte cité, mais y renvoie par un titre, un nom d'auteur, de personnage ou l'expose d'une situation spécifique. " ${ }^{1}$

Cela s'applique sur le livre de Fleutiaux dans les points suivants :

1- Dans sa préface elle évoque le livre célèbre de Bruno Bettelheim qu'elle cite sous son titre anglais «The uses of enchantment » qui est traduit vers le français sous le titre «Psychanalyse des contes de fées ».

2- Après cela elle parle de Marc Soriano, le philosophe français, né au Caire et qui était spécialiste des contes de

${ }^{(1)}$ Tiphaine Samoyault, L'Intertextualité ,op.cit.,p.35.

Vol. 39 
Perrault. Elle fait allusion à son livre titré «Les contes de Perrault, culture savante et traditions populaires » paru en 1968.

3- Toujours dans la même préface elle parle des contes de Perrault et ceux des frères Grimm, quelque chose que nous trouvons normale puisqu'elle présente dans cette préface sa réécriture de ces contes. Nous remarquons qu'elle cite plusieurs titres des contes de Perrault y inclus le seul conte des Grimm qu'elle a refait «Blanche Neige ». On lit donc ces titres : La Belle au bois dormant, Le Petit Poucet, .... Tout de même on rencontre d'autres titres des contes en vers et en prose du même auteur qu'elle n'a pas réécrit. Elle dit :

«Un jour Riquet à la houppe, Les fées, et Le chat botté me diront sans doute ce qu'ils ont à me dire. »'

Un autre élément paratextuel où on trouve la référence claire et nette, c'est la quatrième de couverture. On a parlé d'un extrait d'un article de Bertrand Poirot-Delpech. On trouve écrit entre parenthèses «Le Monde ». C'est une autre référence non-littéraire qui vient s'ajouter ici.

${ }^{(1)}$ Pierrette Fleutiaux, Métamorphoses,op.cit.,p.11.

Vol. 39 
Intertextualité et réécriture à travers « Métamorphoses de la reine » de Pierrette Fleutiaux

Si l'on passe au titre et aux intertitres du recueil, on remarque qu'ils ont, eux aussi, un caractère intertextuel, ou au moins, ils soulignent qu'il s'agit d'une réécriture ou inspiration d'autres textes connus par le lecteur. Le titre de l'œuvre fait référence aux œuvres littéraires ayant pour thématique la métamorphose ou le changement profond du caractère du héros, citons à titre d'exemple: "Les Métamorphoses » d'Ovide, et «La Métamorphose » de kafka. Quant aux intertitres, il suffit d'en jeter un regard pour comprendre qu'ils sont en relation avec des contes populaires ou contes de Perrault même s'ils sont détournés. Certains d'entre eux évoquent des personnages-types des contes de Perrault ou bien des lieux qui sont toujours une marque essentielle de ses récits. Barbe bleue, La femme de l'ogre, Les sept géantes et Cendron, sont tous des titres qui nous renvoient directement à l'héritage des contes occidentaux.

De cette façon, la référence accompagne généralement la citation pour en indiquer la source, mais parfois elle apparait seule dans le texte comme c'est le cas chez Fleutiaux. Elle cite surtout dans la préface les titres de plusieurs livres critiques portant sur l'analyse des contes et ses personnages. Cela montre la place qu'occupent les contes dans sa 
bibliothèque et peut aussi aider un lecteur qui veut aller plus loin dans ses lectures sur ces textes.

En somme, les deux types de l'intertextualité les plus marquants chez notre auteure, sont la citation et la référence. Les deux sont des formes explicites qui ne sollicitent beaucoup d'érudition ou d'intelligence de la part du lecteur pour les désigner. Même les textes cités sont trop célèbres pour un lecteur francophone. Mais il sera bon d'insister sur le rôle du lecteur dans le jeu de l'intertexte. C'est sur lui que dépend tout sinon, l'intertextualité n'aura aucun effet. Plusieurs théoriciens ont souligné ce rôle important. Citons Riffaterre qui propose une définition de l'intertextualité tout en la liant au lecteur. Pour lui elle est :

«La perception par le lecteur de rapports entre une cuvre et d'autres, qui l'on précédée ou suivie. Ces autres cuvres constituent l'intertexte de la première. »l

D'après la définition citée là-haut, on peut considérer que le lecteur de Fleutiaux peut remplir cette mission simplement, i.e, la perception de l'intertexte fleutien comme nous venons de voir.

\footnotetext{
${ }^{(1)}$ Michael Riffaterre, "La trace de l'intertexte”, in La pensée, n²15,oct. 1980. cité dans "Introduction à l'intertextualité » de Nathalie Piégay-gros,op.cit.,p.16. 
Cela était le sceau de l'érudition de l'écrivaine, mais aussi, à notre avis, il marque l'intelligence du choix des phrases empruntées aux autres, puisqu'il ne rompt pas, au moins chez la plupart des lecteurs, la continuité de la lecture. On peut lire le texte d'une manière souple sans être obligé d'aller chercher la source de telle ou telle phrase, tout en s'amusant des dits comiques extraits des textes des grands auteurs.

Maintenant et avant de passer aux fonctions de l'intertextualité, il sera convenable de faire une analyse concise de cette pratique dans l'hypotexte de Perrault afin de mettre en évidence le processus de la réécriture chez Fleutiaux et les décalages entre les deux versions.

Est-ce qu'on peut déterminer exactement la provenance des contes de Perrault ou ceux des frères Grimm ? Est-ce que ces auteurs ont totalement inventé ces histoires ou bien il y avait une sorte d'intertextualité avec d'autres textes qui leurs sont antérieurs?

Presque tous les spécialistes des contes nous soulignent qu'ils dépendent des textes oraux anciens. Perrault, Les Grimm et La Fontaine, s'inspiraient des contes anciens populaires. Certains d'entre ces contes ont une source Vol. 39 
déterminée par les chercheurs, d'autres non puisqu'ils dattent d'un passé lointain qui échappe aux recherches des critiques. Mais comme la théorisation et la conceptualisation des termes « intertextualité », «hypertextualité », « réécriture » sont assez récentes, on peut considérer que la présence d'autres textes dans les contes de Perrault par exemple, était dû au hasard. Autrement dit, Charles Perrault lorsqu'il s'inspirait des textes antérieurs, il pratiquait l'intertextualité sans le savoir. C'était juste une admiration des œuvres anciennes qu'il avait l'intention de les faire revivre. En d'autres termes,il appliquait cette pratique à son nouveau texte, sans lui donner son nom par lequel elle est connue aujourd'hui.

Nous ne sommes pas concernés ici de marquer la source et l'histoire de chacun des contes de Perrault qui sont à la base de la réécriture de Fleutiaux, nous allons juste donner une idée bref sur le caractère intertextuel de ces écrits, et du dialogue intertextuel qu'ils ont avec d'autres. Paul Delarue dans son livre «Le conte populaire français» nous montre que les contes occidentaux bien connus comme «Cendrillon» et "Peau d'âne »,... ont des versions multiples européens, 
Intertextualité et réécriture à travers « Métamorphoses de la reine » de Pierrette Fleutiaux asiatiques ou nord-africains. Les analystes ont connu une Cendrillon chinoise qui remonte au IXe siècle. ${ }^{1}$

D'autres contes s'inspirent des histoires orientaux et pharaoniques. On peut dire que tout conte revêt un caractère intertextuel ou un aspect de réécriture. Il dépend généralement d'une reprise d'un symbole, d'un thème, d'un type de personnage afin de refaire un nouveau produit, qui portera un ou plusieurs traits de l'ancien. Ainsi, comme l'hypertexte de Fleutiaux recourt aux jeux intertextuels, celui de Perrault les utilise aussi. Son texte peut être considéré à son tour comme hypertexte à un certain moment. On emprunte ici Pascale Hellegouarc'h en disant :

"Cette intertextualité confirme une matière appartenant à la culture commune, suffisamment dense pour devenir support de déclinaisons et de récupérations plus ou moins parodiques. ${ }^{2}$

C'est la nature riche et magique qui qualifie généralement les contes et les histoires oraux qui aident à la survivance et au renouvellement continuel de ces textes.

${ }^{(1)}$ Paul Delarue, Le conte populaire français, Tome premier,éd.GP Maisonneuve et Larose,1976,p.7.

(1) Lecture $d u$ Petit Chaperon Rouge: didactique de l'intertextualité, Pascale Hellegouarc'h, in Synergies France n ${ }^{\circ}-2010$,pp.79-88,p.79.

Vol. 39 
Pour aborder l'intertexte qui réunit les contes perraldiens à d'autres textes, il suffit de donner l'exemple des trois contes les plus étudiés sur ce point, et qui sont aussi repris par Fleutiaux à savoir: Barbe Bleue, La Belle au bois dormant, et Le Petit Chaperon Rouge.

Ute Heidmann a mené une recherche détaillée sur ce point dans son article qui porte le titre : «Expérimentation générique et dialogue intertextuel: Perrault, La Fontaine, Apulée, Straparola. ${ }^{1}$. Selon son hypothèse, il y a un dialogue intertextuel entre ces trois contes et «Les Métamorphoses» d'Apulée ou son «Ane d'or». Ce roman de l'écrivain latin Apulée se compose de onze livres racontant l'histoire de Lucius transformé en âne par accident et qui ,pour regagner sa forme humaine il doit passer par des épreuves et des aventures tout en racontant au lecteur des histoires telle "Psyché et Cupidon $»^{2}$

Heidmann considère que Perrault s'est inspiré pour chacun des contes cités précédemment d'une épisode de ce

\footnotetext{
${ }^{(1)}$ Ute Heidmann, Expérimentation générique et dialogue intertextuel : Perrault, La Fontaine, Apulée, Straparola, Féeries (en ligne), 8/2011, http://www.feeries.revues.org/777

(2) www.wikipedia.org

Vol. 39 
Intertextualité et réécriture à travers « Métamorphoses de la reine » de Pierrette Fleutiaux roman latin, et parfois aussi il s'inspire des histoires racontées par le héros, lors de ses aventures, comme celle de Psyché.

D'autre part il emprunte certains aspects du recueil de l'écrivain italien Basile* «Lo cunto de li cunti » . Dans «La belle au bois dormant» de Perrault, l'auteur puise quelques éléments du texte de l'écrivain napolitain, comme la mère royale aux appétits d'ogresse. ${ }^{1}$ Ainsi, on peut dire que Perrault, mais aussi les frères Grimm, se sont inspirés des contes européens et des genres déjà existants pour créer de nouvelles histoires adaptées à leurs temps. Nous avons essayé de donner quelques exemples d'intertextualité chez l'écrivain classique parce qu'une analyse détaillée implique une étude à part.

Il nous semble donc que les contes de Perrault dépendent largement du dialogue intertextuel. En effet :

\footnotetext{
*Giambattista Basile, poète et écrivain italien né à Naples. Son œuvre la plus célèbre est le recueil intitulé «Le conte des contes» (Lo cunto de li cunti) et qui est nommé aussi « Pentamerone ».

*Texte rédigé en langue napolitaine publié entre 1634 et 1636 . Il est structuré autour d'un récit-cadre dans lequel sont racontées 49 histoires sur 5 jours. Basile a groupé dans ce recueil, qui est considéré comme le premier livre européen basé totalement sur des contes, des histoires de la tradition orale. Perrault et ensuite les frères Grimm se sont inspiré de ce livre pour des histoires telles : La Belle au bois dormant, Les feés, Hansel et Gretel. Cf.wikipedia.org. *

${ }^{(1)}$ Ute Heidmann, article cité,p.60.
}

Vol. 39 
«Les contes de Perrault recourent à plusieurs intertextes à la fois, en créant des réseaux hautement complexes de strates intertextuelles différentes. C'est cette superposition de différents dialogues intertextuels qui fait de ces textes de véritables palimpsestes. » ${ }^{1}$

Le texte de Fleutiaux peut être considéré comme une parodie des contes traditionnels. Cette pratique, selon Genette, est appliquable à un texte par le biais de plusieurs types. Depuis Aristote, la parodie (parôdia), était un genre littéraire qui est le contraire de l'épopée noble et sérieux. C'était donc un genre narratif qui modifie le texte noble au niveau de son style et de son contenu. ${ }^{2}$

Fleutiaux a donc repris les textes de Perrault ainsi que l'un des contes des frères Grimm, en modifiant le style à certains endroits mais en modifiant l'esprit et le contenu tout de même. On peut considérer que le nouveau texte qu'on a entre les mains est une raillerie du texte de Perrault, ou bien qu'il présente une critique des idées et des jugements qui y trouvent surtout en ce qui concerne le femme.

\footnotetext{
(1) Ibid,p.68-69.

(2) G.Genette,Palimpsestes,op.cit.,p.22.
}

Vol. 39 
Intertextualité et réécriture à travers « Métamorphoses de la reine » de Pierrette Fleutiaux

Ainsi , nous avons trouvé adéquat de souligner les liens intertextuels qui unissent l'hypotexte et l'hypertexte. Nous avons déjà signalé les points d'intertextualité avec d'autres textes en dehors de ceux de Perrault. Mais maintenant, il faut souligner l'intertextualité avec les contes originaux qui peut être présentée à travers certaines formules qui débutent les contes, ou bien à travers des phrases qui se répètent au niveau de la narration.

Les contes de Perrault sont marqués par certaines phrases et certaines expressions qui sont forgées dans la tête $\mathrm{du}$ lecteur. Lorsqu'on rencontre "Il était une fois» on consentit qu'on va lire ou écouter un conte. Christophe Carlier compare ce type de formulette au trois coups qu'on écoute au théâtre et qui marquent le début d'une pièce :

"Comme les trois coups, au théâtre, annoncent l'arrivée des acteurs, la formulette authentifie le conte dont elle balise le déroulement ${ }^{1}$

Presque tous les contes de Perrault qui ont été refaits par Fleutiaux, commencent par «Il était une fois... ». Cette formule engage le récit et marque le début de l'histoire. Chez Fleutiaux, ce n'est pas toujours le cas. Tantôt elle commence

${ }^{(1)}$ Christophe Carlier,La clef des contes, éd. Ellipses,Paris,1998,p.52.

Vol. 39 
son texte par le même début, tantôt elle reformule les phrases et donne à son texte des mots tout à fait différents de ceux utilisés par les conteurs d'autrefois.

Pour suivre l'ordre des nouvelles comme dans le recueil, nous devons commencer par «La femme de l'ogre ». Cette nouvelle ne respecte pas la règle et la première phrase qui y figure c'est :

«La femme de l'ogre n'aime pas préparer la chair, mais elle ne le sait pas. " Une phrase qui annonce une relation d'hypertextualité avec le texte ancien, plutôt qu'une relation d'intertextualité.

Après cela vient l'histoire de «Cendron» qui commence cette fois-ci par la formulette qui est chère au genre du conte, et ces quelques phrases sont à comparer à celles au début de "Cendrillon ou la petite pantoufle de verre » :

«Il était une fois une veuve «Il était une fois un qui épousa en secondes gentilhomme qui épousa en

${ }^{(1)}$ Pierrette Fleutiaux, Métamorphoses,op.cit.,p.15. 
Intertextualité et réécriture à travers « Métamorphoses de la reine » de Pierrette Fleutiaux noces le gentilhomme le plus secondes noces une femme, la frivole et le plus hautain plus hautaine et la plus fière qu'on ê̂t jamais vu. ${ }^{1} \quad$ qu'ont ê̂t jamais vue. ${ }^{2}$

Presque les deux textes partagent les mêmes formulettes. Néanmoins, le texte de Fleutiaux a inversé le genre de l'un des personnages (Gentilhomme vs. Veuve) et veuve vient indiquer ici l'état civil de la femme,mère de Cendron, ainsi que les adjectifs (Fière vs. Frivole). Fleutiaux nous donne un trait de caractère de ce gentilhomme; il n'est pas seulement arrogant, mais il est tout de même léger et il manque de sérieux. Mais elle a eu recours, à peu près, au même style et au même vocabulaire utilisés par Perrault.

Passons à «Le différé de la reine». Cette nouvelle reprend le début bien connu des contes et le reste de la phrase nous fait un clin d'œil qu'on est en train de lire une réécriture de «La belle au bois dormant ». Une petite comparaison est faite dans ce tableau pour montrer la phrase qui annonce chacune des deux histoires :

«Il était une fois un prince et «Il était une fois un Roi et une

\footnotetext{
${ }^{(2)}$ Ibid.,p.53.

${ }^{(3)}$ Charles Perrault,Contes,op.cit.,p.259.
}

Vol. 39 


\begin{tabular}{l|l|l} 
une princesse si amoureux et si & Reine, qui étaient si fâchés de \\
pressés de s'unir qu'on ne & $n$ 'avoir point d'enfants, si \\
saurait le dire."l & $\begin{array}{l}\text { fâchés qu'on ne saurait } \\
\text { dire." } 2\end{array}$
\end{tabular}

Dans «Petit Pantalon Rouge, Barbe Bleue et Notules» Fleutiaux reprend quelques mots et quelques phrases qui tissent une relation intertextuelle avec les deux contes de Perrault mélangés dans cette nouvelle : Le Petit Chaperon Rouge et La Barbe Bleue. Par exemple, on remarque qu'elle reprend des termes archaïques qui marquent la langue des contes tels : mère-grand, chaperon,.... De la même façon elle a réutilisé la phrase : "....petit chaperon rouge qui lui seyait si bien ....»

Des passages sont empruntés littéralement à la Barbe Bleue originale. La description de ce personnage est presque la même sous la plume des deux écrivains. Perrault disait à propos de lui :

«Il était une fois un homme qui avait de belles maisons à la ville comme à la campagne, de la vaisselle d'or et d'argent, des meubles en broderie, et des carrosses tout

\footnotetext{
${ }^{(1)}$ Pierrette Fleutiaux, Métamorphoses,p.85.

${ }^{(2)}$ Charles Perrault, Contes,p.185.
}

Vol. 39 
Intertextualité et réécriture à travers « Métamorphoses de la reine » de Pierrette Fleutiaux dorés ; mais par malheur cet homme avait la Barbe bleue : cela le rendait si laid et si terrible, qu'il n'était ni femme ni fille qui ne s'enfuît de devant lui. "»

A la page 110 du livre de Fleutiaux, on lit les mêmes phrases citées mais on rencontre quelques phrases intercallées dans un dessein de réécriture ironique qui sera étudiée isolément.

Suivant la même manière, PPR essaye de libérer les sept femmes de BB. Elle s'adresse à sa mère et sa grand-mère avec les mêmes phrases utilisées par la femme de BB de Perrault qui demande l'aide de sa sœur Anne. En remplaçant BB par les deux frères de sa femme, la version de Perrault se sert du même dialogue :

\begin{tabular}{|l|l|l|}
\hline "Montez au sommet de la tour & «Monte, je te prie, sur le haut \\
pour voir si BB ne vient point. & de la tour, pour voir si mes \\
Il m'avait promis qu'il serait là & frères ne viennent point ; ils \\
aujourd'hui et si vous le voyez, & m'ont promis qu'ils me me \\
faites-lui signe de se hâter. " & viendraient voir aujourd'hui, et
\end{tabular}

\footnotetext{
${ }^{(1)}$ Charles Perrault,Contes,op.cit.,p.219.

${ }^{(1)}$ Pierrette Fleutiaux, Métamorphoses,op.cit.,p.125.
}

Vol. 39 

se hâter. " ${ }^{1}$

Pour parler de la nouvelle intitulée «Les sept géantes», la réécriture de "Blanche Neige» des Grimm, il faut noter que le début du conte varie d'une version à l'autre et d'une traduction à l'autre. Certaines versions commencent par « Il était une fois» et d'autres non. La nouvelle de Fleutiaux n'adopte pas le formule. De même, le dialogue bien connu qui s'est déroulé entre la reine et son miroir : (Miroir, petit miroir chéri, quelle est la plus belle de tout le pays ?) n'est pas repris de la même façon dans le conte refait, mais il est réutilisé plusieurs fois de manières diverses. C'est-à-dire que le dialogue se répète en faisant référence à des détails de beauté. On se demande devant le miroir parfois de la taille la plus belle, de la voix la plus douce, ou du teint le plus clair du pays.. .

Ainsi cette nouvelle a subi une grande part de la réécriture de façon que des passages importants qui marquent ce récit ont été refait selon le nouveau contexte. On retrouve la même atmosphère du conte des Grimm, quand même l'étude de l'intertextualité dans cette partie sera resteinte.

${ }^{(2)}$ Charles Perrault,Contes,p.226. 
Puisque la septième et la dernière histoire du recueil « Le palais de la reine » n'est pas la réécriture d'aucun conte de Perrault comme c'est cité dans la préface, elle sera exclue de l'analyse intertextuelle. On passe donc à «La reine au bois dormant » qu'est «La belle au bois dormant» de Perrault mais tout à fait transformé. Fleutiaux a repris les personnages : reine, roi, et les enfants Aurore et Petit Jour. Mais en lisant le texte, on retrouve qu'il relève plutôt de l'hypertextualité que de l'intertextualité.

Il est temps de conclure. Le travail de Fleutiaux émane dans son intégralité d'une retransformation des contes de Perrault et des Grimm, cette transformation souligne un décalage avec le texte initial, ce qui est mis en évidence à travers les points choisis de notre analyse. Cependant, il y a avait quelques traits d'intertextualité avec des textes différents $\mathrm{du}$ texte original et d'autres qui sont en relation avec lui. Ces points ne sont pas nombreux, ils sont parfois au début du texte, et parfois ils sont au cœur du texte, et un lecteur qui n'est pas assez cultivé, peut facilement les identifier. Cela peut paraitre normal, parce que l'écrivaine a complètement détourné le texte ancien. Elle a revisité certaines idées, 
certaines situations pour les refaire à son gré. Elle reprend parfois certaines phrases telles quelles, et dans un autre moment elle les détourne soit pour être en accord avec le nouveau contexte, soit dans une intention de ridiculiser certains personnages.

\section{Fonctions de l'intertextualité}

L'autre volet de notre étude sur l'intertextualité dans «Métamorphoses de la reine» porte sur les fonctions de cette pratique dans toute ses formes. Certes, l'allusion, la référence ou la citaion ne sont pas juste des fragments empruntés à un texte et inséré dans un autre, au contraire ils remplissent un certain nombre de fonctions qu'on peut énumérer comme suit :

1-Marc Eigeldinger dans son livre «Mythologie et intertextualité » nous montre que la première fonction de l'intertextualité est une fonction "référentielle et stratégique $»^{1}$. Cela veut dire que la citation, par exemple, peut « se référer » à un autre texte antérieur au texte qu'on est en train de lire. Tout de même elle peut se référer à un autre

(1) Marc Eigeldinger, Mythologie et intertextualité,éd. Slatkine,1987,p.16. Vol. 39 
Intertextualité et réécriture à travers « Métamorphoses de la reine » de Pierrette Fleutiaux domaine artistique : le cinéma, la peinture,... D'autre part, elle nous donne une idée sur la référence culturelle ou idéologique d'un personnage de telle ou telle œuvre, ce qui peut nous aider à le caractériser et à bien comprendre son statut dans le roman ou le recueil. Cela est bien remarquable chez Fleutiaux. Dans «Cendron », le héros et la reine quand ils recourent à des textes littéraires qui ne sont pas français parfois, nous donnent des éclairements sur leur culture, leut univers référentiel culturel, ils ont des points en commun que l'auteure nous met en évidence. D'un autre côté, les deux frères de Cendron quand ils citent Molière, cela émanait d'une astuce d'humour de Fleutiaux, et c'est pourquoi peut être qu'elle a choisi cette pièce pour en citer quelques vers et non pas les vers d'un écrivain sérieux. De cette paradoxe entre les deux citations et leur contexte, celle de la Reine et Cendron d'une part, et celle de ses deux frères de l'autre , l'écrivaine fait référence au niveau culturel de chacun des protagonistes.

2-La deuxième fonction, toujours selon M.Eigeldinger, est « transformatrice et sémantique ». Il dit à ce propos : 
"Il ne s'agit pas de reproduire à l'état brut le matériau d'emprunt, mais de le métamorphoser et de le transposer. »"

Il partage le même avis d'Antoine Compagnon qui considère que l'intertextualité est une relation entre deux texte, c'est-à- dire deux système sémiotyques. Un auteur reprend une phrase ou un extrait d'un texte tel qu'il est, mais l'utilise en lui donnant une nouvelle valeur, pour exprimer une idée différente de celle exprimée dans le texte d'origine. ${ }^{2}$

Ainsi, le fait d'extraire des dits de Dante, de Molière, de La Fontaine ou même de Jules César, donne une nouvelle dimension à un personnage ou à une situation au trame des nouvelles de notre recueil.

3-L'intertextualité peut aussi avoir une fonction "éthique ou rhétorique». A chaque allusion, citation ou référence résultent deux choses: premièrement on fait attention à la culture de l'auteur de l'œuvre, et deuxièmement on forme un lien entre auteur/narrateur et lecteur. Ce dernier peut tout de même enrichir ses propres savoirs à travers l'intertextualité, en cherchant dans les livres, il peut tracer un extrait donné. On retrouve cela dans le texte de Fleutiaux

\footnotetext{
${ }^{(1)}$ Ibid.

(1) Antoine Compagnon, La seconde main ou le travail de la citation,op.cit.,p.5657.
} 
Intertextualité et réécriture à travers « Métamorphoses de la reine » de Pierrette Fleutiaux dans certains cas d'intertexte. Lorsqu'elle recourt à La Fontaine ou Molière par exemple, elle cite des passages bien connus du lecteur occidental. Même quand on parle de Dante, il n'est pas difficile d'en savoir la source, ou bien on peut chercher à qui appartient cette phrase mise entre guillemets.

4-« La fonction ludique » est la quatrième des fonctions intertextuelles. Pour expliquer ce point, on peut se renvoyer au livre de Michel Picard intitulé "La lecture comme jeu», il dit :

«Aussi, que la lecture puisse être comparée, voire assimilée à un jeu particulier s'est imposé avec plus ou moins d'évidence à quantité de lecteurs et à plus d'un critique. »"

En partant de la citation précédente, on peut dire que si la lecture peut être considérée comme un jeu, cette métaphore s'impose d'une manière plus claire lorsqu'il s'agit d'un livre à caractère intertextuel. C'est-à-dire que l'allusion ou la citation invitent le lecteur à décoder le texte, à identifier l'intertexte, de cette manière il participe à ce jeu avec l'auteur. Plus le lecteur est avisé et cultivé, plus la perception de l'œuvre est plus riche et profitable. Or, cela se voit clairement aussi

${ }^{(1)}$ Michel Picard, La lecture comme jeu, éd. de Minuit, Paris, 1986,p.11. Vol. 39 
lorsqu'il s'agit d'une parodie par exemple. On imite un écrivain, un modèle afin de provoquer des effets comiques, mais ici la fonction ludique peut revêtir un aspect critique, parce qu'elle reconsidère un stéréotype ou un cliché ou même un certain type de personnage. Fleutiaux a, de sa part, critiqué et reconstruit la relation homme/femme dans son nouveau récit.

5-La dernière fonction de cette pratique est une "fonction de justification», ou une «fonction argumentative ». Un intertexte peut être argumentative à deux niveaux : il peut argumenter une certaine attitude de la part de l'un des personnages d'un roman, pièce,..., ou bien il peut argumenter et justifier l'opinion de l'auteur du livre. Nous reprenons parfois les paroles d'autrui pour montrer qu'il y a quelqu'un d'autre qui renforce ce que nous disons, et ainsi on est sûr de notre avis. Une citation peut donc être parfois un signe de doute.

Pour résumer, l'intertextualité joue un rôle important dans un texte. Cela varie selon sa forme. Elle est tantôt signe d'authentification ou de documentation, et tantôt elle met en évidence l'arrière-plan culturel de l'écrivain. Tout en plus, elle 
Intertextualité et réécriture à travers « Métamorphoses de la reine » de Pierrette Fleutiaux peut présenter aux lecteurs l'un des personnages d'une œuvre, c'est ainsi qu'elle nous aide à le caractériser, à comprendre ses hantises, ses obsessions et son savoir. ${ }^{1}$ Si l'on parle d'une allusion implicite, cela peut rafraîchir la mémoire du lecteur et le pousse à participer à un jeu intéressant avec l'auteur et le narrateur. Après tout cela, l'intertextualité reste au cœur de toute littérature.

\section{Contes mélangés et mise en abyme}

Les contes sont des textes façonnables. Cela veut dire que le schéma narratif, les personnages et les motifs des contes sont des éléments dociles qui peuvent être repris sans cesse pour créer de nouveaux produits. En profitant de cet avantage, Fleutiaux nous semble avoir recourt à plusieurs procédés de la réécriture des contes dont l'une est «les contes mélangés ». Cette pratique comme son nom l'indique, consiste à amalgamer dans une même histoire des personnages issus des contes différents. Quelquefois ils conservent le rôle et les caractéristiques qu'ils avaient dans le conte original, et dans d'autres cas, ils peuvent subir des opérations de changement et de métamorphose dûs au processus de la réécriture par lequel ils passent.

\footnotetext{
(1) Nathalie Piégay-Gros, Introduction à l'intertextualité, op.cit.,p.76.
} 
De cette façon, les nouvelles de Fleutiaux ont repris les personnages des célèbres contes mais dans un autre décor. Chacun des héros des contes de Perrault se trouve sous la plume de Fleutiaux, arraché à son récit et implanté dans un autre. Et c'est aussi l'un des atouts des ces récits. Puisque si l'on essaye d'appliquer ce procédé à d'autres récits moins connus, le processus ne sera pas éfficace.

Ainsi en feuilletant les contes réécrits, nous allons voir "La femme de l'ogre » qui a regagné un statut plus important, nous allons la voir rencontrer «Le Petit Poucet» qui n'a pas de nouvelle qui porte son nom dans le recueil. Quant à La Barbe Bleue, il cède sa place dans les contes de Perrault pour rejoindre notre «Petit Pantalon Rouge» mais tout en conservant ses caractéristiques physiques et morales.

Nous pouvons donc constater que Pierrette Fleutiaux n'a pas seulement eu recours à l'intertextualité, ou bien a détourné ou déconstruit les contes de Perrault, mais aussi elle a superposé plusieurs récits anciens les uns sur les autres. La rencontre qu'elle a fait entre les personnages des différentes histoires et des intrigues peut procréer un effet parfois comique et toujours ludique, parce que ce mélange savoureux 
Intertextualité et réécriture à travers « Métamorphoses de la reine » de Pierrette Fleutiaux

des contes peut enthousiasmer le lecteur et l'initier à entrer dans ce jeu avec l'écrivain.

On peut dire que l'œuvre de Fleutiaux a pu former avec les contes de Perrault, mais aussi avec d'autres textes une relation qui prend la forme de «réseau». Ce réseau a plusieurs typologies selon Tauveron ${ }^{1}$ :

1-Un réseau intertextuel qui est constitué du texte citant et des textes cités.

2-Un réseau hypertextuel qui regroupe des textes dérivés d'un texte source. ${ }^{2}$

Les deux types des réseaux sont présents dans le texte de Fleutiaux. Le premier relève de la notion d'intertextualité et de ses différentes formes. Et le deuxième envoie à toutes les catégories du détournement d'un texte: reformulation, adaptation, réécriture.....

Certainement, ces réseaux enrichissent le texte. En outre, cette réunion des deux types, fait une sorte de remueméninges au lecteur et rompt son horizon d'attente* puisqu'il

(1) Catherine Tauveron, Comprendre et interpréter le littéraire à l'école: du texte réticent au texte proliférant, éd. Repères, 1999, p.19. ${ }^{*}$

(1) Réseaunances: aspects de l'intertextualité dans une lecture en réseau, Marc Parayre, Skholê, hors-série I,141-148,p.142.

*Ce terme a été étudié par Hans Robert Jauss dans son livre "Pour une esthétique de la réception". Dans cette théorie , H.R. Jauss axe son étude sur le "sujet Vol. 39 
va lire une autre chose tout à fait différent de ses contes d'enfance.

\section{Le Petit Chaperon Rouge : une réécriture dans la réécriture ?}

Dans la première nouvelle du receuil intitulée « $L a$ femme de l'ogre », l'heroïne se trouve à un certain moment dans une situation de contage. La femme de l'ogre avant de coucher les petites ogrelettes, elle leur raconte l'histoire du «Petit Chaperon Rouge». Si Fleutiaux a fait une réécriture pour ce conte qu'est le «Petit Pantalon Rouge », il s'agit ici d'autre chose. La femme en racontant l'histoire la détourne. Elle essaye de faire apprendre à ses ogresses de choisir ce que l'on mange, de manger proprement, elle leur apprend que tout ce que leur père, l'ogre, fait n'est pas correct. Brièvement, elle essaye de les aider à devenir des petites filles. Ce conte était l'un de ses armes dans son combat, mais toujours en vain. Elle n'a pas pu changer la réalité que ses petites sont des ogresses et qu'elles vont désormais agir de cette façon suivant leurs instincts :

consommateur » ou le lecteur et non pas l'auteur. Il insiste sur la réception d'une œuvre et non pas sa production. L'horizon d'attente c'est ce qu'un lecteur attend d'un livre ou d'une œuvre littéraire. Une bonne œuvre dite artistique, doit tromper et surprendre le lecteur, elle doit s'opposer à son horizon d'attente déjà existant. Vol. 39 
Intertextualité et réécriture à travers « Métamorphoses de la reine » de Pierrette Fleutiaux

«(...) et lorsqu'elles sont toutes au lit, entreprend de leur raconter un conte.

-Il était une fois une petite fille de village. Sa mère et sa mère-grand, qui en étaient folles, lui avaient fait faire un petit chaperon rouge...

-Rouge comment? s'écrient les ogrelettes.

-Coquelicot,dit la mère pensant aux bords des chemins et aux fleurs qu'elle aime.

-Non! disent les enfants.

-Fraise, dit la mère pensant aux surprises de ses promenades dans les champs.

-Non! crient les enfants.

Et comme la femme égarée se tait un instant, les enfants aussitôt se mettent à crier.

-Sang de bouf, sang de taureau, sang de bouf, sang de taureau.

\section{(...)}

-Sa mère et sa mère-grand lui avaient fait faire un petit chaperon rouge sang. Un jour, sa mère ayant cuit et fait des galettes...

-Pouah,pouah, crient les enfants.

-Quoi ? dit la mère. 
-(...) pas des galettes,pas des galettes.

$-(\ldots)$

$-(. .$.$) des belettes, des belettes. { }^{1}$

$-(\ldots)$

Cette histoire que raconte la femme est enchâssée ou mise en abyme dans la nouvelle. Et il est à signaler que ce terme «la mise en abyme » a été utilisé par André Gide pour la prmière fois en 1893. Mais Lucien Dällenbach lui confère une nouvelle signifaication d'œuvre dans l'œuvre ou de narration seconde incluse dans une narration première et entretenant avec elle une relation d'analogie thématique. ${ }^{2}$

La présence de ce récit dans le récit principal a une connotation. Claude de la Grenadière souligne :

"Raconter le Petit Chaperon Rouge est devenu, dans ce récit, une joute entre femme et enfants, joute qui a fait surgir chez elles, et entre elles, l'enjeu même du conte. (...) si la femme de l'ogre s'égare, les ogrelettes, elles, sentent avec le récit leurs appétits croître dans leur corps, jusqu'à convoiter celle-là même qui raconte. (...) il lui faut cependant

\footnotetext{
${ }^{(1)}$ Pierrette Fleutiaux, Métamorphoses de la reine,op.cit.,p.30-31.

${ }^{(1)}$ Christian Angelet, Onze études sur la mise en abyme, , Belgique,1980,p.9.
}

Vol. 39 
Intertextualité et réécriture à travers « Métamorphoses de la reine » de Pierrette Fleutiaux

décider: lutter encore avec elles pour maintenir la voix du conte ou s'abandonner au réel de la voracité pulsionnelle. »"

La femme de l'ogre a choisi de raconter ce conte aux ogrelettes, en l'utilisant comme moyen pour échapper au réel. Elle espérait sentir qu'elle est mère de filles normales qui écoutent toujours ce que leur mère dit, et comprennent le sens de l'interdit. Mais malheureusement la femme se trouve toute seule devant l'ogre son mari et ses ogresses ayant le même appétit que lui. Même lorsqu'elle raconte l'histoire elle la détourne, de sa part mais après l'objection des ogrelettes, elle reprend le récit pour le refaire selon leur goût et leur volonté. Elle n'a eu rien à leur égard et elle n'a pas pu rien changer. Enfin et pour clore le récit elle propose cette fin :

«-Taisez-vous, dit la mère, il est temps de se coucher et voici la fin du conte. "Mère-grand,dit le loup, que vous avez de jolies dents pointues.

-C'est pour mieux te manger, petit loup, et disant ces mots cette méchante petite fille se jeta sur le pauvre petit loup et le dévora. $»^{2}$

${ }^{(2)}$ Claude de la Grenadière, Encore un conte? Le Petit Chaperon Rouge à l'usage des adultes, éd. L'Harmattan,1996,p.192.

${ }^{(1)}$ Pierrette Fleutiaux, Métamorphoses de la reine, op.cit.,p.34-35.

Vol. 39 
La femme se laisse aller à la volonté de ses ogrelettes à la fin de l'histoire, et renonce à la lutte lorsqu'elle se rend sûre qu'elles ne sont que "les miniatures de leur père » selon l'expression de Bettina L. Knapp. ${ }^{1}$

Enfin, le conte original du «Petit Chaperon Rouge » a été repris maintes fois. Une fois on le lit totalement modifié, et une autre fois on le trouve enchâssé dans un autre récit. Les ogrelettes et leur mère nous offrent de nouvelles propositions en ce qui concerne les événements du conte. Il nous reste à signaler que certains analystes considère que la mise en abyme est l'un des types de l'intertextualité. Marc Eigeldinger, voit que les quatre types de l'intertextualité sont comme suit : la citation, l'allusion, le pastiche et la parodie, et finalement la mise en abyme. ${ }^{2}$ Peut être il met en évidence cette hypothèse puisque la mise en abyme peut avoir une certaine analogie avec l'intertextualité parce que c'est une relation de présence d'un texte dans un autre. Mais en tout cas, c'est une technique qui a laissé une trace dans le texte de Pierrette Fleutiaux ,et sur laquelle on a dû attirer l'attention.

\section{Conclusion}

\footnotetext{
${ }^{(2)}$ Bettina Knapp,Pierrette Fleutiaux,op.cit.,p.117.

${ }^{(2)}$ Marc Eigeldinger, Mythologie et intertextualité,op.cit.,p.14. 
Intertextualité et réécriture à travers « Métamorphoses de la reine » de Pierrette Fleutiaux

Ainsi, on a remarqué que "Métamorphoses de la reine » de Pierrette Fleutiaux est marqué par l'abondance des allusions, des citations et références à des écrivains et des œuvres anciennes. Le jeu de l'intertextualité et de la réécriture est intéressant dans la mesure où il contribue à construire un nouveau texte, mais en même temps à déconstruire un autre. Et cela est fait tout au long du texte même au niveau paratextuel.

En plus, il est important de signaler que l'œuvre littéraire ne peut être étudiée indépendamment de son contexte social. La littérature influe la société mais aussi reflète ses valeurs et sa façon de penser. Donc,Fleutiaux a essayé à travers les fragments intertextuels qu'elle a choisis, et à l'aide de la technique de la mise en abyme de réévaluer certains points de vue concernant les héroïnes de ses nouvelles et par conséquent la femme en général. En faisant cela, elle a critiqué l'œuvre de Perrault ainsi que certaines valeurs de son temps.

D'autre part, elle a émaillé son texte de certaines phrases empruntées à d'autres textes pour donner à son œuvre une dimension ironique et amusante. On peut dire que l'intertextualité et la réécriture dans ce recueil s'inscrivent 
dans une stratégie esthétique et idéologique de Fleutiaux. On considère donc qu'elle a fait un amalgame de l'intertextualité et de l'écriture subvertive pour imposer un nouveau regard à la situation de la femme et celui de l'homme ainsi que leur relation, tout en se servant des textes antérieurs connus par tout le monde. Et les éléments intertextuels choisis, ont contribué à véhiculer son message au lecteur. 
Intertextualité et réécriture à travers « Métamorphoses de la reine » de Pierrette Fleutiaux

\section{Bibliographie sélective}

\section{I- $\quad \underline{\text { Le corpus : }}$}

-Pierrette Fleutiaux, Métamorphoses de la reine, prix Goncourt de la nouvelle 1985,Ed.Gallimard,collection folio $\mathrm{n}^{\circ} 2183,1984$

-Charles Perrault, Contes, le livre de poche $\mathrm{n}^{\circ} 21026$, 2006

\section{II- Bibliographie de l'auteure :}

1- Aux éditions Actes sud:

BONJOUR, ANNE mars 2010

LA SAISON DE MON CONTENTEMENT - mars 2008

LES ETOILES A L'ENVERS - New York, photo roman

LES AMANTS IMPARFAITS - 19 août 2005

DES PHRASES COURTES, MA CHERIE - $\quad$ septembre 2001 Actes Sud BABEL (avril 2003)

HISTOIRE DU GOUFFRE ET DE LA LUNETTE, nouvelles, 1976, Actes Sud Babel

(cf. La fuite en douce, film de Michel Deville)

\section{2- Aux éditions Gallimard:}

L'expédition, roman, 1999, repris en folio $n^{\circ} 3405$

ALLONS NOUS ETRE HEUREUX?, roman, 1994, repris en Folio n²890

SAUVEE ! , nouvelles, 1993, repris en Folio n²719

Vol. 39 
NOUS SOMMES ETERNELS, roman, 1990, prix Femina, repris en Folio n²413

WE ARE ETERNAL, 1994, Little Brown, New York

HISTOIRE DU TABLEAU, roman, (prix Marie-Claire Femmes), Julliard, repris en Folio $\mathrm{n}^{\circ} 2447$

HISTOIRE DE LA CHAUVE-SOURIS, roman, 1975, (avant propos de Julio Cortázar), Julliard, repris en Folio, ${ }^{\circ} 2445$

\section{3- Aux éditions Julliard:}

LA FORTERESSE, nouvelles, 1979

HISTOIRE DU GOUFFRE ET DE LA LUNETTE, nouvelles, 1976

\section{4- Pour la jeunesse :}

TRINI A L'ILE DE PAQUES, Gallimard, Folio Junior, 1999

TRINI FAIT DES VAGUES, Gallimard, Folio Junior, 1997, Prix des jeunes lecteurs de Valenciennes

LA MAISON DES VOYAGES (en collaboration avec Alain Wagneur), Gallimard, Page Blanche, 1997, Prix"Lire au Collège"

MON FRERE AU DEGRE X, 1995, L'école des Loisirs

LE CHEVAL FLAMME, (sur Delacroix) Calmann-Lévy et Réunion des musées nationaux

\section{5- Autre travaux et publications :}

L'os d'Aurochs, nouvelle Editions du Chemin de Fer -sous la direction de Renaud Buenerd Neuf leçons de littérature

Essais

(Michel Butor, Chloé Delaume, P.Fleutiaux, Claudine Galea, Hédi Kaddour, Richard Millet, Oliver Rohe, Antoine Volodine, Cécile Wajsbrot)
Editions
Thierry
Magnier
2007

avec La Maison des Ecrivains et France Culture

Vol. 39 
Intertextualité et réécriture à travers « Métamorphoses de la reine » de Pierrette Fleutiaux

Projet initié par Marguerite Gateau et Cécile Wajsbrot

Leçons prononcées le 25 juin 2006 à la Bibliothèque nationale de France et diffusée le 13 août 2006 sur France Culture

\section{III- Ouvrages critiques consultés :}

- Anne-Claire Gignoux, La récriture: formes, enjeux, valeus autour du nouveau roman, éd., Presses Paris Sorbonne, 2003

- Antoine Compagnon, La seconde main ou le travail de la citation, éd. du Seuil, Paris, 1979

- Catherine Tauveron, Comprendre et interpréter le littéraire à l'école: du texte réticent au texte proliférant, éd. Repères, 1999

- Christian Angelet, Onze études sur la mise en abyme, , Belgique, 1980

- Christophe Carlier,La clef des contes, éd. Ellipses,Paris,1998

- Claude de la Grenadière, Encore un conte? Le Petit Chaperon Rouge à l'usage des adultes, éd. L'Harmattan, 1996

- Gérard Genette, Palimpsestes, La littérature au second degré, Seuil, 1982

- Julia Kristeva, Sémiotiké, Recherche pour une sémanalyse, in Intertextualité mémoire de la littérature, Tiphaine Samoyault, éd., Nathan, Paris, 2001

- Marc Eigeldinger, Mythologie et intertextualité,éd. Slatkine, 1987

- Michael Riffaterre, "La trace de l'intertexte”, in La pensée, $\mathrm{n}^{\circ} 215$,oct. 1980

- Michel Picard, La lecture comme jeu, éd. de Minuit, Paris, 1986

- Michel Schneider, Voleurs de mots, Paris, Gallimard, 1985

- Nathalie Piégay-Gros ,Introduction à l'intertextualité, , éd., Dunod, Paris, 1996

- Paul Delarue, Le conte populaire français, Tome premier,éd.GP Maisonneuve et Larose,1976

- Intertextualité mémoire de la littérature, Tiphaine Samoyault, éd., Nathan, Paris, 2001 


\section{IV- Sitographie :}

- www.larousse.fr/encyclopedie.

- www.jdlf.com.lesfables/livres.

- www.site-moliere.com/pieces/bourg.204.htm.

- www.wikipedia.org.

- www.operone.de/dante.

- www.lepoint.fr.

- $\quad$ www.feeries.revues.org/777. 